\title{
Numerical Analysis of the Physical Properties of the Flow of Molten Polymers in Grooved Profiles
}

\author{
Sérgio Rodrigues Morais Jr., Diego Alves de Miranda \\ Department of Mechanical Engineering, University of the Region of Joinville-UNIVILLE, Joinville, Brazil \\ Email: sergiojunao516@gmail.com
}

How to cite this paper: Morais Jr., S.R. and de Miranda, D.A. (2019) Numerical Analysis of the Physical Properties of the Flow of Molten Polymers in Grooved Profiles. Open Access Library Journal, 6: e5179.

https://doi.org/10.4236/oalib.1105179

Received: January 11, 2019

Accepted: January 22, 2019

Published: January 25, 2019

Copyright $\odot 2019$ by author(s) and Open Access Library Inc.

This work is licensed under the Creative Commons Attribution International License (CC BY 4.0).

http://creativecommons.org/licenses/by/4.0/

\begin{abstract}
There is a wide field of research on the physical properties of non-isothermal pseudoplastic fluid flow. These researches help to avoid processing failures in the fields of thermoplastics. The present work presents the effects of flow inside cavities with groove profiles. For the simulations, the Ansys fluent software was used, considering steady-state flow without incompressible fluid slip. The government equations used in the simulations were mass conservation, momentum conservation and energy conservation. In order to discretize the equations, the finite volume method was used. Similar processes are seen in the thermoplastic injection industry which justifies the intention to identify the aspects of the process which hinders thermal exchange and favors the formation of undesirable physical properties in the injected parts. The implemented viscosity model was validated with the literature and consequently allowing the application of a complete analysis of the physical properties in the flow of molten polymers.
\end{abstract}

\section{Subject Areas}

Computer Engineering, Experimental Physics, Fluid Mechanics, Mathematical Analysis

\section{Keywords}

Thermoplastics Injection Process, Non-Newtonian Fluids, Numerical Simulation, Non-Isothermal Flow

\section{Introduction}

According to Piatti and Rodrigues (2005) [1], polymers are relatively inexpen- 
sive materials for a variety of applications, from surgical materials to toys, to each polymer, to a different application, to a different raw material, and to a different process. According to Cruz and Pacheco (2004) [2], the technological progress of humanity has an incremental character, that is, technologies complement each other and generate other technologies, with that polymers is not different. However, these have a much more elementary character. According to Zorzi and Perottoni (2004) [3], the versatility of polymers not only implies different physicochemical characteristics, but also impacts in the volume and geometry of manufactured products whose manufacturing processes require prior and well-defined specifications, most of the complex geometries have sudden changes of volume in different sections of the product. According to Miranda et al. (2017) [4], the process of injection of thermoplastics increasingly requires monitoring and control, and above all, obtaining previous parameters in order to know design and processing restrictions, since small variations can have considerable costs for manufacturers, therefore the need to use prototypes [5]. Like prototypes, simulations serve to save resources by researching and developing new processes and products.

Specifically, polymer processing simulations in injection molds are composed of three steps: filling, cooling and packing. According to Miranda (2018) [6], filling a mold consists of melting the polymer and injecting it into a type of shell in the final shape of the product; the flow of the polymer into the mold depends on the injection mold temperature, polymer viscosity, injection temperature, and injection speed or pressure, among other characteristics. During the filling, the polymer begins to lose heat to the mold and cools down, making the temperature change, which impacts the viscosity of the fluid; however, the greatest part of the cooling occurs when the mold is already completely filled [7]. Another very relevant study was made by Oumer et al. (2009) [8] that developed a model that characterized the suspension flow of fibers in bidimensional molds; in another study, Chen et al. (2002) [9] implemented a solution that determined the temperature, pressure and velocity distributions at various stages of the fluid advance in the injection-compression process.

In this context, the present article demonstrates the procedure of obtaining physical data on polymer flows fused in wells with grooves parallel to their trajectory through simulations, whose conclusions extend to a large part of the injection processes; such results reinforce already existing works and confirm intuitive beliefs within the framework of the simulation.

\section{Methodology}

\subsection{Validation}

To validate any simulation it is necessary to create a safe environment, it is known that Koh et al. (2004) [10] obtained relevant and replicable results in flat geometric models. The two-dimensional control volume used by this author are two horizontal parallel lines called upper and lower walls and two parallel vertic- 
al lines whose space between them define inlet and outlet, defining a channel without volumetric restriction, but with planar restriction according to Figure 1 .

According to Figure 1, the control volume is a rectangular section measuring height of $1 \mathrm{~cm}$ and a length of $10 \mathrm{~cm}$, its dimension parallel to the plane is undefined, the simulated material is the POM or polyoxylmethylene, which is also the material chosen for the simulation of this article. Another similarity between Koh and the object of study is found in its boundary conditions, which are permanent regime (time independence) and incompressible flow (defined by equations that result in no variation of fluid density). The properties used are illustrated in Table 1.

Since $T_{0}$ is the input temperature of the fluid in the channel, $T_{w}$ is the temperature of both channel walls, $U_{0}$ is the uniform input velocity of the fluid, $\rho$ is the specific mass of the fluid, $\boldsymbol{k}$ is the thermal conductivity of the POM and $C_{p}$ is the specific heat of the POM, it is necessary to emphasize that although the incoming pressure is not revealed according to Table 1, its development is totally possible since the problem provides an inlet velocity.

\subsection{Adapted Governing Equations}

In the simulation that described by the present article, the flow follows some contour conditions, which are stationary, incompressible and laminar flow, this impacts on the government equations that in fact are conditions that must be respected in the simulation in order that the results obtained in the simulations are correspond to reality. Government equations are always described in partial differential forms, since there are multiple other variables that depend on such equations due to the two-dimensional aspect of the problem, they also mathematically define the previously specified boundary conditions as shown below, their variables are found in Table 2.

According to Table 2, all variables are in the international system. Equations (1)-(5) represent the mass balance, amount of movement, energy balance, shear rate and the viscosity model, respectively.

$$
\begin{gathered}
\frac{\partial\left(\rho \mu_{j}\right)}{\partial x_{j}}=0 \\
\frac{\partial\left(\rho \mu_{j} \mu_{i}\right)}{\partial x_{j}}=\frac{\partial}{\partial x_{j}}\left(\eta \frac{\partial\left(\mu_{i}\right)}{\partial x_{j}}\right)-\frac{\partial(P)}{\partial x_{i}}+\frac{\partial}{\partial x_{j}}\left(\eta \frac{\partial\left(\mu_{j}\right)}{\partial x_{i}}\right) \\
\frac{\partial\left(\rho C_{P} \mu_{j} T\right)}{\partial x_{j}}=\frac{\partial}{\partial x_{j}}\left(k \frac{\partial T}{\partial x_{j}}\right)+\eta \gamma^{\dot{2}} \\
\dot{\gamma}=\sqrt{\frac{1}{2}\left(\frac{\partial \mu_{i}}{\partial x_{j}}+\frac{\partial \mu_{j}}{\partial x_{i}}\right)^{2}} \\
\eta=m \dot{\gamma}^{n_{1}-1} e^{a T}
\end{gathered}
$$




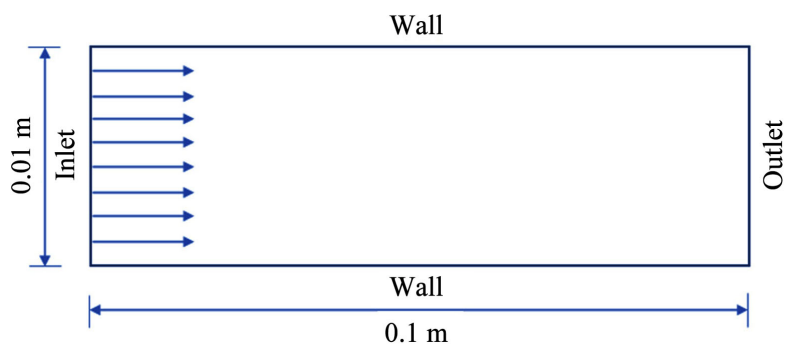

Figure 1. Scheme of the problem, adapted from Koh et al. 2004 [10].

Table 1. Initials conditions used in effect of temperature and inlet velocity on the flow of a non-newtonian fluid.

\begin{tabular}{cc}
\hline Variables & Values \\
\hline$T_{0}$ & $493 \mathrm{~K}$ \\
$T_{w}$ & $440 \mathrm{~K}$ \\
$U_{0}$ & $0.1 \mathrm{~m} / \mathrm{s}$ \\
$\rho$ & $1198 \mathrm{~kg} / \mathrm{m}^{3}$ \\
$k$ & $0.125 \mathrm{~W} /(\mathrm{m} \cdot \mathrm{K})$ \\
$C_{p}$ & $3182 \mathrm{~J} /(\mathrm{kg} \cdot \mathrm{K})$ \\
\hline
\end{tabular}

Table 2. Used variables.

\begin{tabular}{ccc}
\hline Variables & Meaning & $\mathrm{Unit}$ \\
\hline $\boldsymbol{\rho}$ & Density & $\mathrm{Kg} / \mathrm{m}^{3}$ \\
$\boldsymbol{\mu}$ & Velocity & $\mathrm{m} / \mathrm{s}$ \\
$\boldsymbol{\eta}$ & Apparent viscosity & $\mathrm{Pa} . \mathrm{s}$ \\
$\boldsymbol{P}$ & Pressure & $\mathrm{Pa}$ \\
$\boldsymbol{x}_{\boldsymbol{j}}$ & Axial direction & $\mathrm{m}$ \\
$\boldsymbol{x}_{\boldsymbol{i}}$ & Perpendicular direction & $\mathrm{m}$ \\
$\boldsymbol{T}$ & Specific heat & $\mathrm{KJ} / \mathrm{kg} \cdot \mathrm{K}$ \\
$\boldsymbol{k}$ & Temperature & $\mathrm{K}$ \\
$\dot{\boldsymbol{r}}$ & Thermal conductivity & $\mathrm{W} / \mathrm{mK}$ \\
$\boldsymbol{m}$ & Shear rate & $\mathrm{Pa}{ }^{-1}$ \\
\hline
\end{tabular}

According to Koh et al. (2004), such a viscosity model is called first-order moldfow, and consists of power law model with the addition of a temperature-dependent coefficient. According to Koszkul and Nabialek (2004) [11], the model has a drop in shear rates, but most of the time the shear rates are high enough, and can affect the numerical method used [12].

\subsection{Control Volume}

The simulation of flow in finned volumes is interesting because there is a signif- 
icant increase in the amount of heat exchange of the fluid with the wall by the simple increase of area of thermal exchange. Another interesting aspect is the sudden change of direction of the fluid, since this also increases the thermal exchange by increasing the convective coefficient of the fluid, specifically in the polymer industry, this aspect is important for the high rate of change of properties through thermal exchange in plastics. Figure 2(a) and asymmetric finite tube, represented in Figure 2(b), use the same variables, boundary conditions and considerations to evaluate the influence of the fins in the fluid flow.

The simulated volumes are $10 \mathrm{~cm}$ in length and have hollow fins, the first order moldflow viscosity model and the initial conditions identical to Koh shall be used, since the main objective is to compare how such flow affects the properties of the fluid, the equations of government stands for both. It is noticed that the only difference is the way the fins are arranged, since the spacing between the fins is the same of the distance between them, which enabled a symmetrical literal alternation, a simple way of explaining the profile of Figure 2(b) is basically to move the fins of Figure 2(a) above the center line $5 \mathrm{~mm}$ to the left, while the fins below the center line $5 \mathrm{~mm}$ to the right. For both simulations, were used meshes of square elements, with dimensions of $0.25 \mathrm{~mm}$, resulting in elements of area of $0.0625 \mathrm{~mm}^{2}$.

\subsection{A Dimensional Processing}

It is a process that is intrinsic to the variety of volumes of the flow profiles applicable for analysis, such methodology allows to analyze a same flow profile without having to perform simulations of larger volumes, becoming an important standard to present results, since such result can be extended to much larger or smaller volumes provided that the shape of the control volume, material and other contour conditions are maintained, however, the micro and macroscopic characteristics of the material must be respected, especially for very large or small volumes, the process consists of presenting the data as a percentage or as the name suggests, dimensionless, from this, the values of Equations (6)-(9) could simply be substituted and such values would be valid, the variables used are: Dimensionless Velocity $Y^{*}=\frac{\vec{Y}}{h}$, Instant Velocity in one given point $(\vec{U})$, Entrance Velocity $\left(u_{O}\right)$, Dimensionless Temperature $(\theta)$, Instant Temperature in one given point $(\vec{T})$, Fluid Entrance Temperature $\left(T_{O}\right)$, Wall Temperature $\left(T_{w}\right)$, Dimensionless Height $\left(Y^{*}\right)$, Instant Height $(\vec{Y})$, and Height $(h)$. Velocity:

$$
U^{*}=\frac{\vec{U}}{u_{o}}
$$

Temperature:

$$
\theta=\frac{\vec{T}-T_{w}}{T_{o}-T_{w}}
$$

Geometry: 


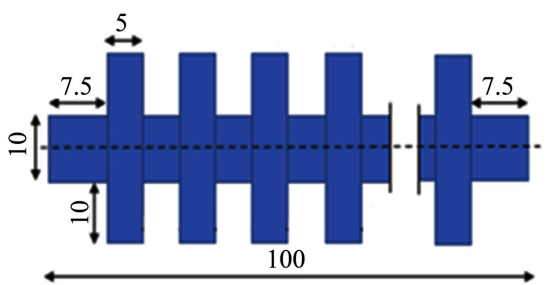

(a)

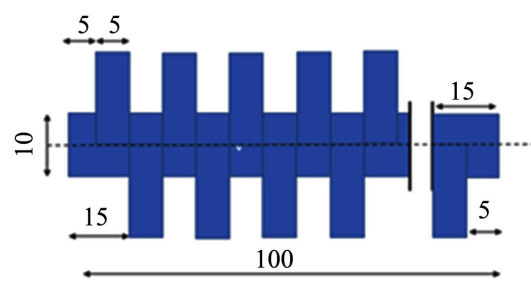

(a)

Unit: $\mathrm{mm}$

Figure 2. Profiles used, symmetrical (a) and asymmetrical (b).

$$
Y^{*}=\frac{\vec{Y}}{h}
$$

It is necessary to emphasize that the notation of vector does not have this meaning, but representing instantaneousness or punctuality, being the left equalities the outputs that can be plotted.

\section{Results}

\subsection{Validation of Simulations}

It was necessary to replicate the Koh simulation to validate the executed properties, since the material used is the POM. The simulations were recreated under the same proposed conditions and help to compare the results. Recreating the results of dimensionlessness of height as a function of dimensionless velocity, temperature and viscosity prove that the properties can be extended to other control volumes; such properties can be expressed graphically according to Figures 3(a)-(c) respectively.

The simulation proposed by Koh establishes equal temperatures in both walls in the value of $440 \mathrm{~K}$ and the fluid in the initial temperature of $490 \mathrm{~K}$, due to the symmetrical characteristic of the control volume used for validation, the height-to-temperature rate also presents symmetry from the center of the channel.

Since the instantaneous velocity increases almost linearly with the distance from the wall, such velocity is directly impacted by the viscosity that varies as a function of the temperature, the expected behavior is observed due to the decrease of the viscosity with the increase of the temperature, as indicated Figure 3 (b), which matches the graphical description of the viscosity in Figure 3(c), in which there is a decrease in viscosity due to the lower temperatures, reflecting on an increase in velocity, similar to an envelope function of viscosity.

\subsection{Graphical Analysis of Contours}

The analysis of the graphs matches the expected validation and respects the values established by Koh et al. showing that the parameters of the simulation are correct; such parameters can be applied to any desired profiles based on the same boundary conditions. As previously mentioned, it was tried to preserve the entrance temperatures and walls in a way that facilitates the comparison of the 
results of thermal exchange between the profiles, it is necessary to emphasize that a certain subtlety is expected in the difference between the results of the symmetrical and alternating profiles, as seen in Figure 4.

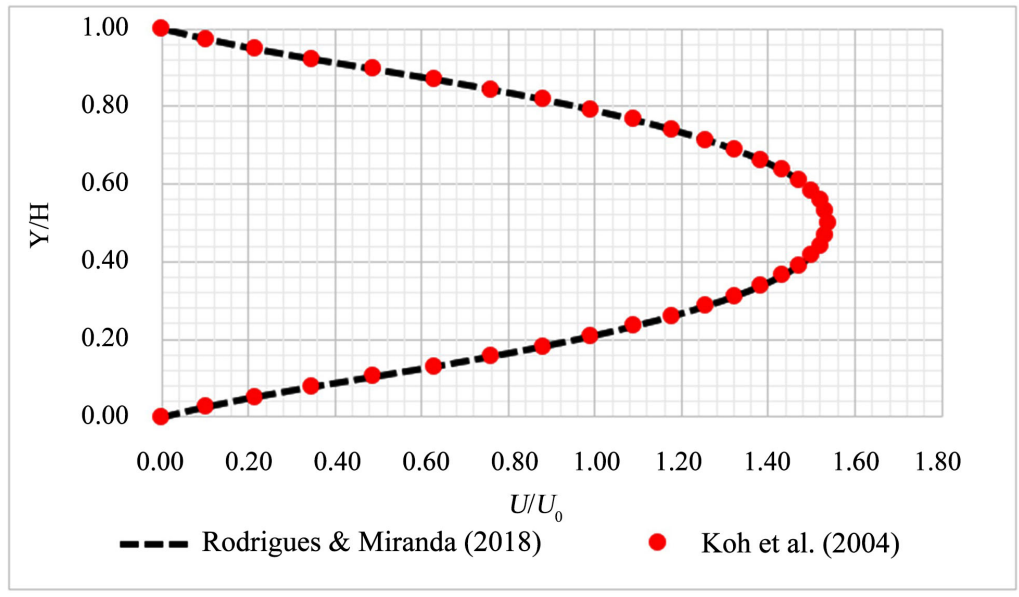

(a)

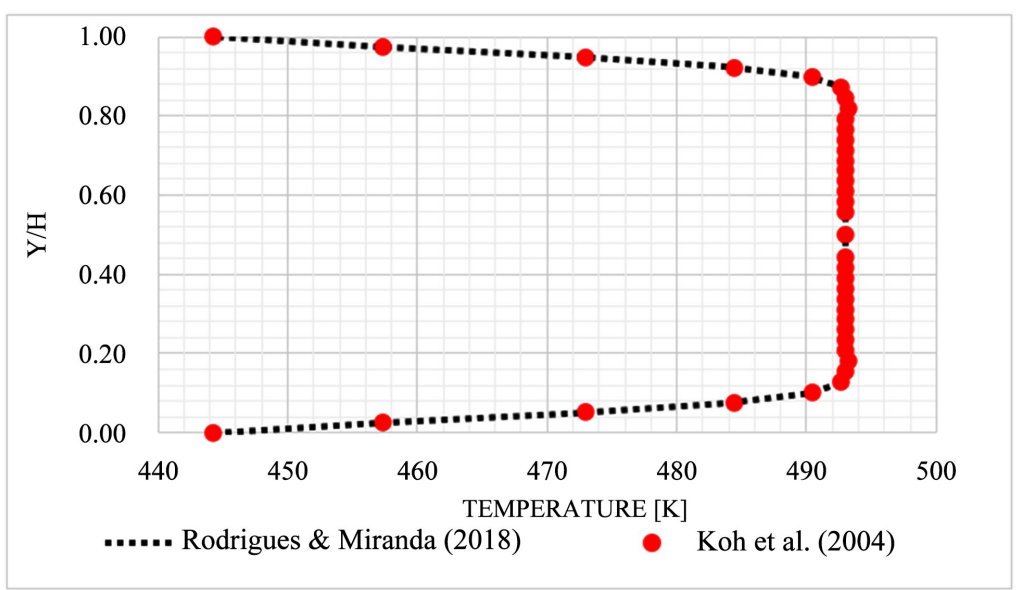

(b)

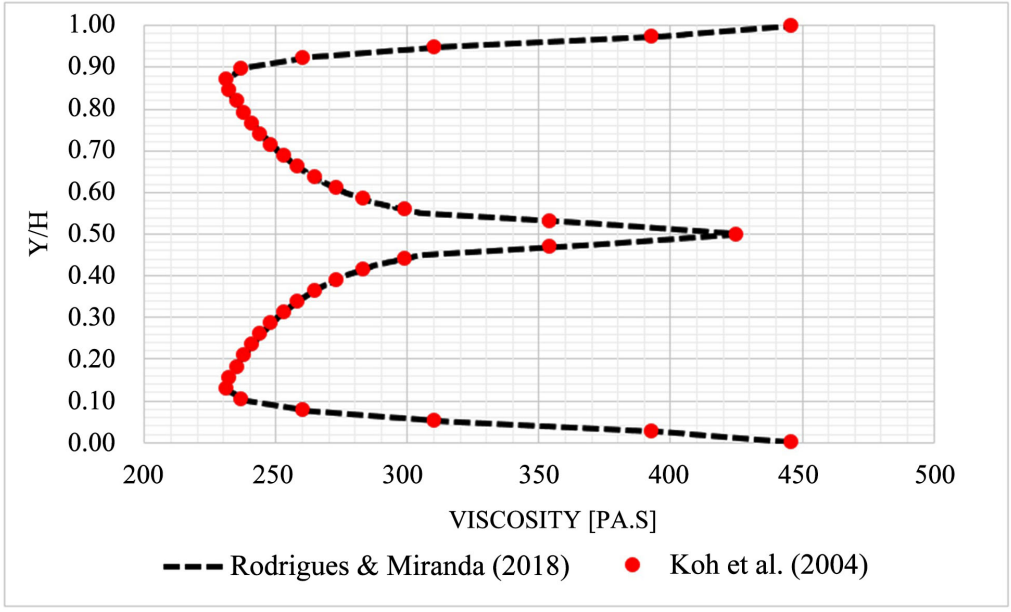

(c)

Figure 3. Comparison of validation data obtained. 


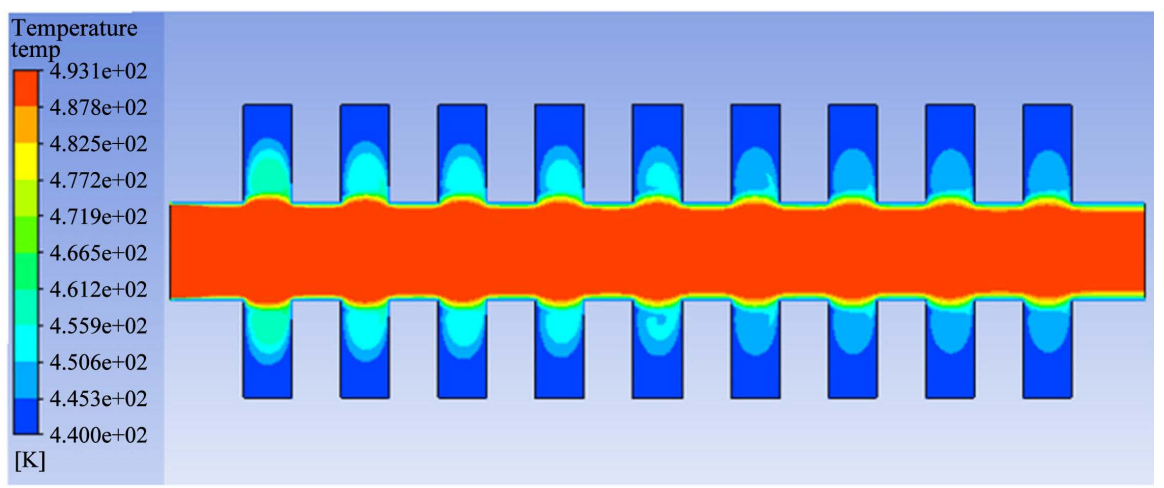

(a)

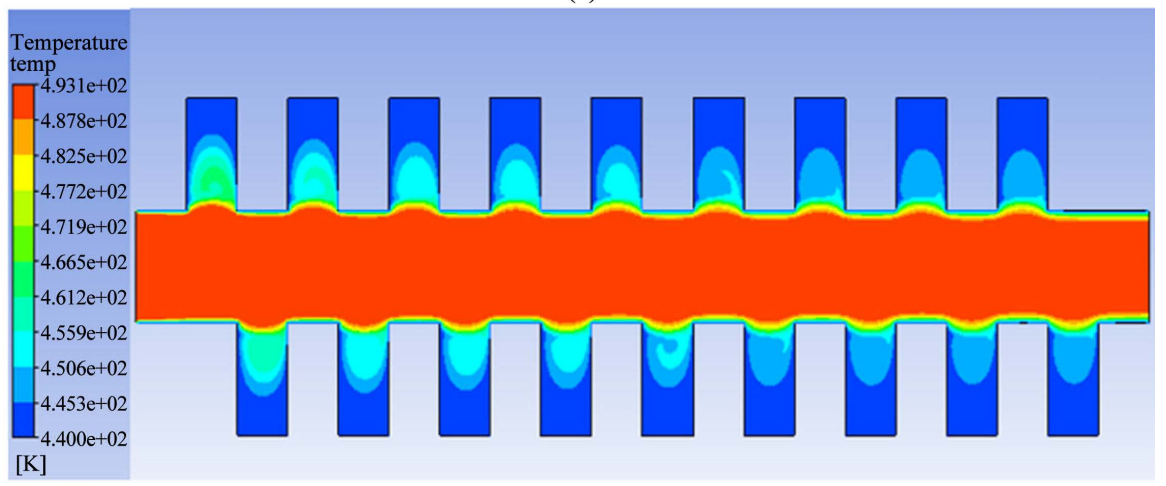

(b)

Figure 4. Temperature contours in both profiles.

Figure 4(a) shows a more linear behavior in thermal losses because in the symmetric finned profile shown, the fluid exchanges heat with two fins simultaneously, while in the alternating profile in Figure 4(b) the fluid exchanges heat with only one, which results in a naturally more erratic contour. Unfortunately, it is not possible to perceive great changes comparing the contours coupled with the temperature of the fins, being necessary a different approach for the determination of application. Observing Figure 5, we can see the same phenomenon, in which there is a small variation of viscosity between the profiles (a) and (b).

Because viscosity its inverse relation to temperature, increases with its fall, we can see this phenomenon in the extremities of the fins, we also observe point increases of viscosity in the longitudinal center line about $2.5 \mathrm{~mm}$ before the entrances of the fins, this is due to the sudden change of direction causing balanced and symmetrical losses of kinetic energy observed in Figure 5. It is observed that there is an increase in viscosity at the end of the fins of both profiles due to the low temperature observed, this is a reflection of the fin profile, which hinders the circulation of POM in this region.

Figure 5(a) shows a similar effect to Figure 5(b), in which there are areas of increase of viscosity, however, as expected the profile generated is different, creating punctual increases that resemble zigzag movements, this occurs due to the asymmetry of the tube, in which the load losses are not symmetrical along the channel, phenomena reinforced in Figure 6. 
The fluid velocity contour in Figure 6(a) oscillates according to the lower viscosity zones of the center line, which in turn are due to sudden cross-sectional increases, the speed variation profile accompanying the flap profile, as previously noted with the viscosity analysis, the role of the cross section in the instantaneous velocity drops is remarkable. While the velocity contour in the alternating finned profile of Figure 6(b) shows a more subtle oscillation of the velocity in the center line that accompanies the profile of the tube, such graphs reveal that, as expected, the velocity of the fluid at the ends of the however, do not give us notion of the path traveled by the fluid inside the fins, for this, it is necessary to reveal the current lines, shown in Figure 7.

According to the details A and B shown in Figure 7, which are details of the current lines in the first fins, a greater accumulation of lines in DET B is noticed, obviously due to the accumulation of material, which in turn is due to the fact that in the first fin in DET. B there is only one fin to be filled while in DET A. There is less fluid since there is another fin being filled simultaneously.

\subsection{Numerical Analysis of the Outputs of Both Profiles}

The procedure used for this analysis consists of capturing all one thousand values of velocity and temperature and the viscosity of both profiles and generating 6 averages according to Equations (9), (10) and (11), the results of which are presented in Table 3; the comparison of these combined analyses of the contours allows a reliable interpretation of the results.

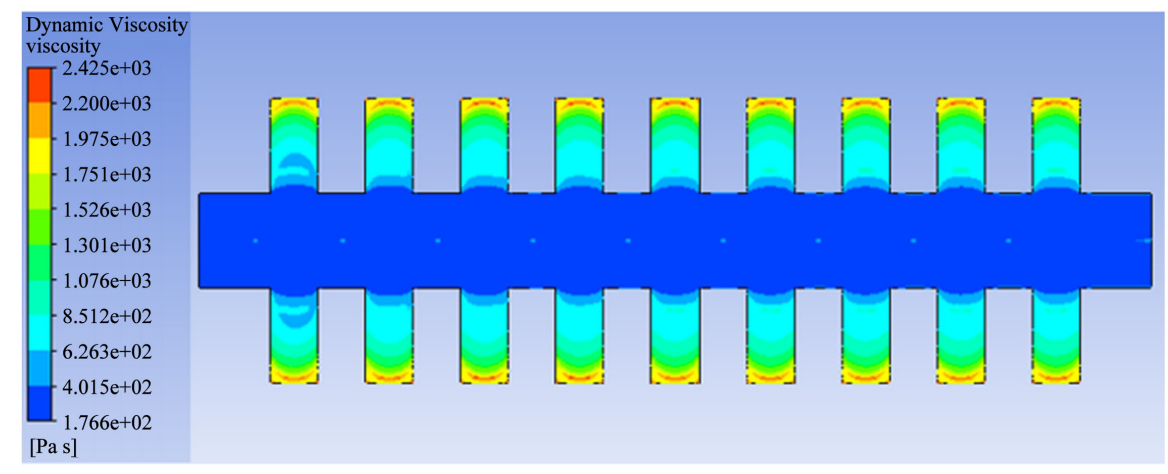

(a)

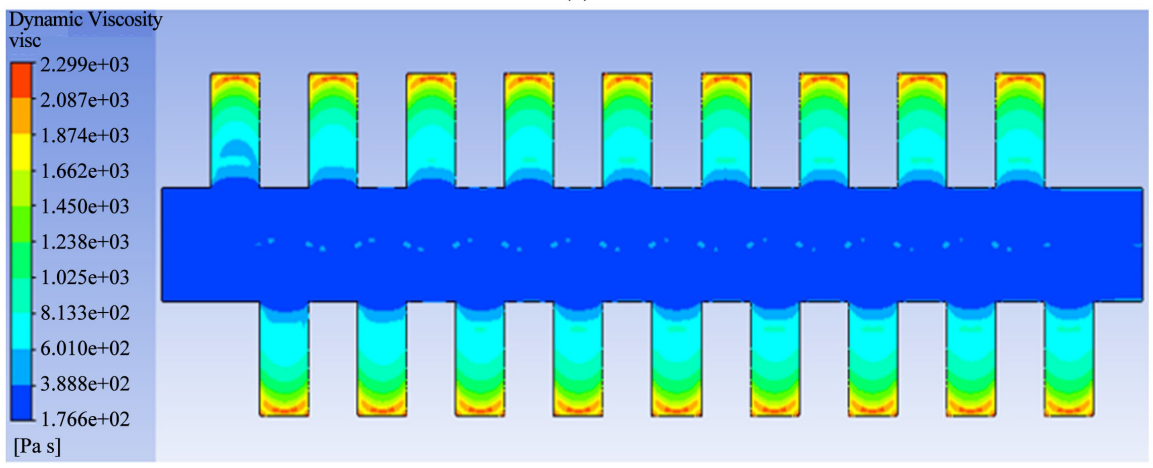

(b)

Figure 5. Viscosity contour in the different regions of both profiles. 


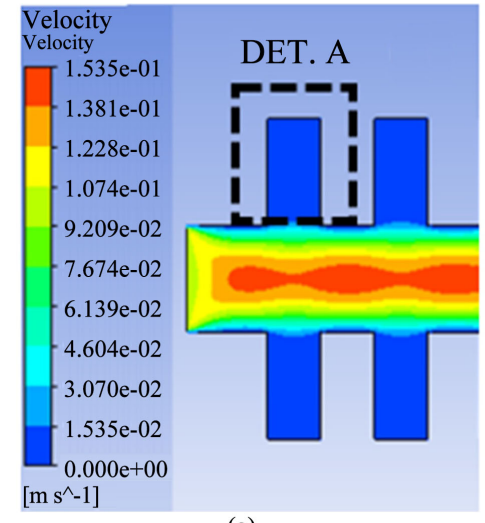

(a)

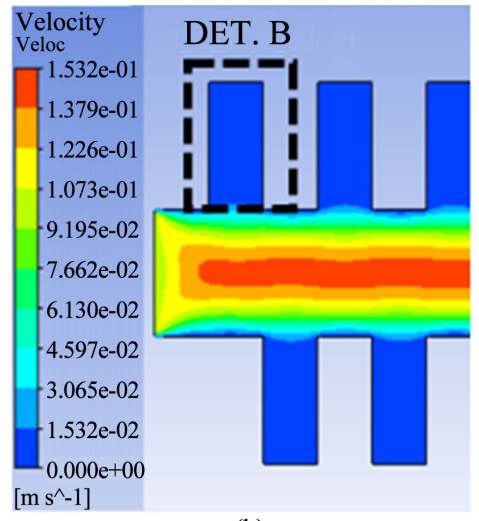

(b)

Figure 6. Velocity contours distributed in both profiles.

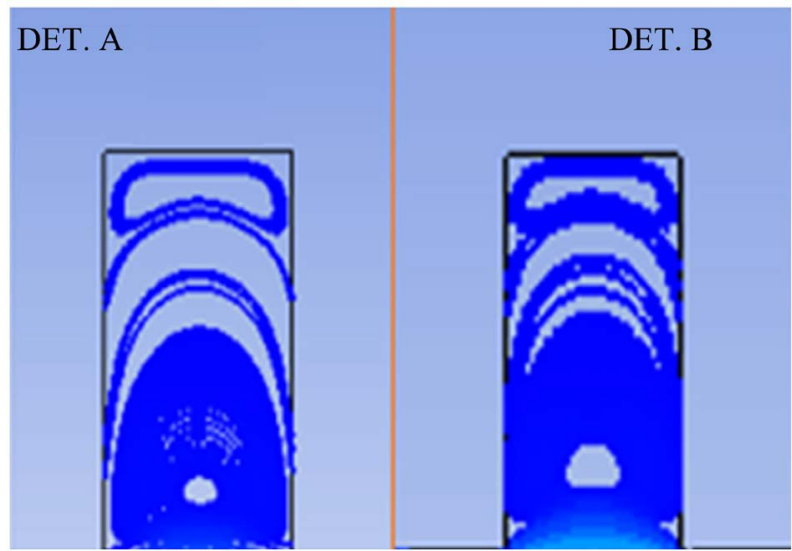

Figure 7. Stream lines. (a) Detail of the symmetrical part; (b) Detail of the asymmetrical part.

Table 3. Data comparison in the outlet.

\begin{tabular}{ccc}
\hline & Symmetrical Profile & Asymmetrical Profile \\
\hline $\bar{U}^{*}$ & 0.997846959342852 & 0.997901247169831 \\
$\bar{\theta}$ & 0.910915955226416 & 0.9107665978312 \\
$\bar{\eta}$ & 278,877 Pa.s & 278,130 Pa.s \\
\hline
\end{tabular}

$$
\begin{aligned}
& \overline{\vec{v}}=\frac{\sum v}{1000} \\
& \bar{\theta}=\frac{\sum T}{1000} \\
& \bar{\eta}=\frac{\sum \eta}{1000}
\end{aligned}
$$

According to the values extracted from Table 3, it can be seen that the value of the mean dimensionless velocity of the asymmetric profile is $0.0000544 \%$ greater than that the symmetrical profile, while the mean of the dimensionless temperature of the symmetrical profile is $0.14 \%$ the mean apparent viscosity of 
the profile is $0.26 \%$ in the symmetrical profile, which reveals that not always average temperatures translate into larger average viscosities macroscopically, since the viscosity model used leads also considering that the shear rate of the fluid that varies from point to point. According to Figure 7, the temperature decrease in these zones is due to the existence of vortices that increase the thermal exchange in the region, cooling the fluid and increasing the viscosity, which also slows down the fluid, this phenomenon also makes difficult the exchange thermal with the central regions, forming a kind of thermal insulation, an effect that is accentuated in regions of lower fluid density where there are areas of lower pressure, as the case with the finely symmetrical profile, on the other hand, the low fluid density in the fins also causes an increase in viscosity that causes more load losses, whereas this effect is smaller in the alternating profile by attenuation of the increase of viscosity in the fins, minimizing the load losses.

Consistent with the numerical analysis on the outputs of both profiles, a small variation of applicability is observed, while the symmetrical profile conserves the temperature better, the alternating profile conserves the fluid flow and its kinetic energy better, a much greater impact on temperature than on speed is observed, obviously, studies with larger fins and stretches of longer length with other fluids are necessary to verify the extension of these phenomena.

\section{Conclusion}

The objective of this work was to report on the steps of the parameters research, establishment of the scenarios, simulation and analysis of the simulation results of two different profiles in a permanent and incompressible regime. All steps were completed and the results were presented. It was also possible to validate the viscosity model used by Koh et al. and apply it to asymmetric and symmetric finned profiles, which, according to the analysis, showed different applications in the case of filling and cooling of polyoxymethylene, with the asymmetric profile being more suitable for molds whose quality was a priority, since such a profile favored better filling of the mold, while the symmetrical profile would have a greater applicability in thermal energy saving, since it seemed to lose less heat to the mold during the process. However, for the confirmation of the observations, there is a need for more studies and battery simulations in order to more clearly contrast these differences among the results and also to verify the ranges of occurrence of these phenomena for other fluids

\section{Conflicts of Interest}

The authors declare no conflicts of interest regarding the publication of this paper.

\section{References}

[1] Piatti, T.M. and Rodrigues, R.A.F. (2005) Plastics: Characteristics, Uses, Production and Environmental Impact. 1st Edition, UFAL, Maceió. (In Portuguese) 
[2] Cruz, C.H.B. and Pacheco, C.A. (2018) Knowledge and Innovation: Brazil's Challenges in the Twenty-First Century. 1st Edition, IFI, Campinas. (In Portuguese)

[3] Zorzi, J.E., Perottoni, C.A. and Jornada, J.A.H. (2004) Low-Pressure Injection Molding of Complex Parts of Advanced Ceramic Produced with Fine Powder. Cerâmica, 50, 202-208. https://doi.org/10.1590/S0366-69132004000300006

[4] Miranda, D.A., Nogueira, A.L., Sacchelli, C.M. and Dreschler, M.F. (2017) Computational Simulation of Thermoplastic Injection: Comparison of Fall-Type Tools. IX Brazilian Congress of Manufacturing Engineering, Joinvile, 26 July 2017, 1-12. (In Portuguese) https://doi.org/10.26678/ABCM.COBEF2017.COF2017-1320

[5] Gavira, M.O. (2003) Computational Simulation as a Knowledge Acquisition Tool. Masters Dissertation, University of São Paulo, São Paulo.

[6] Miranda, D.A. (2018) Influence of Mesh Geometry and Mesh Refinement on Mathematical Models of Thermoplastic Injection Simulation Tools. IOSR Journal of Mechanical and Civil Engineering, 15, 38-44.

[7] Hassan, H., Regnier, N., Le-Bot, C. and Defaye, G. (2010) 3D Study of Cooling System Effect on the Heat Transfer during Polymer Injection Molding. International Journal of Thermal Sciences, 49, 161-169.

https://doi.org/10.1016/j.ijthermalsci.2009.07.006

[8] Oumer, A., Ali, A.M.S. and Mamat, O. (2009) Numerical Simulation of Fibre Orientation in Simple Injection Molding Processes. International Journal of $\mathrm{Me}$ chanical and Mechatronics Engineering, 9, 361-367.

[9] Chen, S.C., Chen, Y.C., Peng, H.S. and Huang, L.T. (2002) Simulation of Injection-Compression Molding Process, Part 3: Effect of Process Conditions on Part Birefringence. Advances in Polymer Technology, 21, 177-187. https://doi.org/10.1002/adv.10024

[10] Koh, Y.H., Ong, N.S., Chen, X.Y., Lam, Y.C. and Chai, J.C. (2004) Effect of Temperature and Inlet Velocity on the Flow of a Nonnewtonian Fluid. International Communications in Heat and Mass Transfer, 31, 1005-1013. https://doi.org/10.1016/j.icheatmasstransfer.2004.05.010

[11] Koszkul, J. and Nabialek, J. (2004) Viscosity Models in Simulation of the Filling Stage of the Injection Molding Process. Journal of Materials Processing Technology, 157-158, 183-187. https://doi.org/10.1016/j.jmatprotec.2004.09.027

[12] Miranda, D.A., Cristofolini, R., Corazza, E.J., Santos, G.J. and Amaral, C.E. (2018) Comparison of Mathematical Methods to Obtain Concentration and Temperature of Newtonian Fluids in Tubular Reactors. Open Access Library Journal, 5, 1-8. https://doi.org/10.4236/oalib.1104329 